\title{
Improved Methods to Determine Optimal Currents in Electrical Impedance Tomography
}

Ping Hua, Member, IEEE, Eung Je Woo, John G. Webster, Fellow, IEEE, and Willis J. Tompkins, Fellow, IEEE

\begin{abstract}
Our electrical impedance tomography (EIT) system uses the optimal current method to inject currents and the regularized Newton-Raphson algorithm to reconstruct an image of resistivity distribution. The optimal current patterns, however, are a function of the unknown resistivity distribution, thus they cannot be obtained analytically. In this paper we developed iterative methods to derive the optimal current patterns through iterative physical measurements. We also developed direct methods to first determine the resistance matrix of a resistivity distribution through a set of experimental measurements, then used the singular value decomposition (SVD) to obtain the optimal current patterns. In both the iterative and direct methods, we injected a complete set of current bases and stored the measured voltage responses. This permitted iterative reconstruction techniques to operate on the stored data without requiring lengthy data taking from the object. This reduced the effects of motion artifacts. We concluded that the direct methods have superior performance as compared to the iterative methods in both optimal current and voltage generation. We studied three sets of current bases: Fourier, diagonal, and neighboring. The Fourier-based method produced most accurate results but required multiple current generators. The diagonal-based method produced slightly less accurate but comparable results using the simple hardware of a single current generator.
\end{abstract}

\section{INTRODUCTION}

$\mathbf{E}$ LECTRICAL impedance tomography (EIT) is a new imaging technique that uses electrical measurements to obtain an object's resistivity distribution. It provides information about an object that is different from that obtained by conventional techniques such as X-ray computed tomography (CT), magnetic resonance imaging (MRI), ultrasonic imaging, and microwave imaging. Bhat [10] summarized the clinical applications of EIT in various physiological systems including the cardiovascular system, respiratory system, and gastrointestinal system. Lytle and Dines [9] described EIT applications in geophysical prospecting.

An EIT data-collection system has a certain amount of measurement error including analog and digital electronics error and quantization error. The measurement error contaminates some of the useful information in the measured data,

Manuscript received May 18, 1991; revised March 13, 1992. This work was supported by the National Science Foundation under Grant EET-8714648.

P. Hua was with the Department of Electrical and Computer Engineering University of Wisconsin, Madison, WI 53706. He is now with the Applied Research Group, Siemens Gammasonics, Inc., Hoffman Estates IL 60195.

E. J. Woo was with the Department of Electrical and Computer Engineering University of Wisconsin, Madison, WI 53706. He is now with the Department of Biomedical Engineering, College of Medicine, Kon Kuk University, Choongbuk 380-701, Korea.

J.G. Webster and W.J. Tompkins are with the Department of Electrical and Computer Engineering, University of Wisconsin, Madison, WI 53706. IEEE Log Number 9201733. depending upon both signal and measurement error levels. This limits the accuracy of image reconstruction under any reconstruction algorithm. Different data-collection methods of injecting currents and measuring the voltages affect the signalto-noise ratios (SNR's) in the measured data.

Isaacson [8] proposed a data-collection method that provides maximal distinguishability. Let $\boldsymbol{R}(\rho)$ denote the resistance matrix for original resistivity distribution $\rho, \boldsymbol{R}(\hat{\rho})$ for the estimated resistivity distribution $\hat{\rho}$. The voltage difference for a given current pattern $I$ is

$$
\boldsymbol{V}_{0}-\boldsymbol{V}_{1}=[\boldsymbol{R}(\rho) \boldsymbol{I}-\boldsymbol{R}(\hat{\rho}) \boldsymbol{I}]=[\boldsymbol{R}(\rho)-\boldsymbol{R}(\hat{\rho})] \boldsymbol{I}=\boldsymbol{D} \boldsymbol{I}
$$

where $D=\boldsymbol{R}(\rho)-\boldsymbol{R}(\hat{\rho})$. The current patterns that maximize the norm of the voltage difference for a unit current, $\left\|\boldsymbol{V}_{0}-\boldsymbol{V}_{1}\right\| /\|\boldsymbol{I}\|$, are defined as the optimal current patterns. The voltage difference is an important term in the updating of resistivity estimation [7], whose maximization can accelerate the convergence of a reconstruction algorithm and minimize the effects of measurement error. Isaacson [8] concluded that the optimal current patterns are the eigenvectors corresponding to the largest eigenvalues of matrix $D$. Note that we limit our study to resistance measurement, and the results can be extended to capacitance measurement.

For the acquired current and voltage data, we can use reconstruction algorithms to obtain the resistivity distribution. There are many reconstruction algorithms for static images including backprojection, perturbation, double constraint, and modified Newton-Raphson algorithms. Yorkey et al. [12] compared these methods and concluded that the modified Newton-Raphson algorithm converges the fastest with the least error. We used the regularized reconstruction algorithm by Hua et al. [6] which improved the performance of the modified Newton-Raphson algorithm by integrating a priori information into the reconstruction algorithm. Fig. 1 shows the flow chart of the algorithm. We first guess an initial resistivity distribution $\rho^{k}$, and use the finite element method (FEM) [7] to calculate its voltage response $f\left(\rho^{k}\right)$ for a given current distribution. We then compare the calculated voltage $f\left(\rho^{k}\right)$ with the measured voltage $V_{0}$ from the real object to see if the mean squared error $\Phi$ is small enough. If it is, we stop and display the resistivity distribution. Otherwise, we update the resistivity distribution by $\Delta \rho^{k}$ according to the given formula until the number of iterations reaches a preset value. In order to avoid local minimum problems, it is useful to start with an educated guess. Note that $D$ is a function of the calculated resistivity distribution; therefore, we update the optimal current 


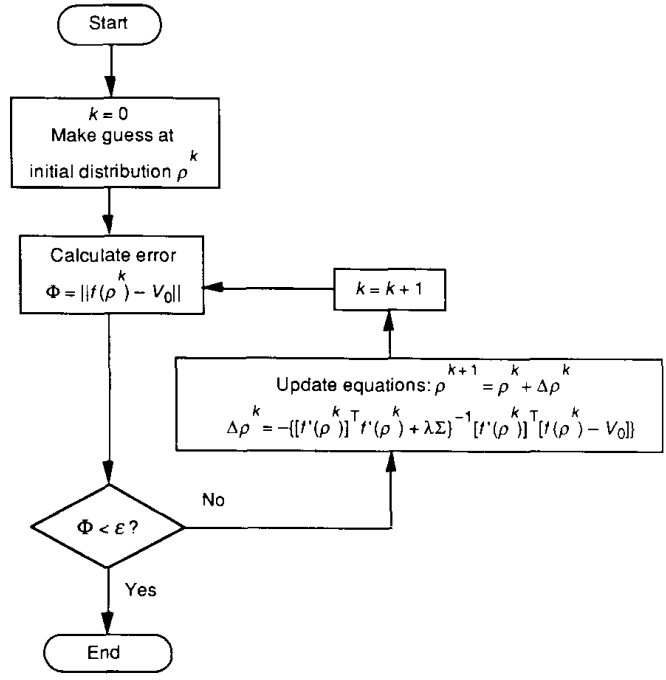

Fig. 1. The modified Newton-Raphson algorithm performs $k$ iterations to minimize the mean-squared error $\Phi$ between the voltages from the real object $V_{0}$ and $f\left(\rho^{k}\right) . f^{\prime}\left(\rho^{k}\right)$ is the Jacobian matrix, $\lambda$ is a smoothing parameter, and $\Sigma$ is a positive definite matrix corresponding to the prior information used.

patterns every few iterations with respect to the new resistivity distribution, and repeat the procedure.

The injection of an optimal current pattern requires multiple current generators. We used a 32-electrode data-acquisition system which is composed of 32 current generators, a voltmeter, and a personal computer (Macintosh II). The current sources are gain-controlled so that they can provide arbitrary current distributions. The voltmeter can be multiplexed to any of the 32 electrodes and is composed of buffers, amplifiers, a demodulator, and filters. The computer controls the currentinjection and voltage-measurement method.

Equation (1) shows that the $D$ matrix is a function of the unknown resistivity distribution, thus its eigensystem, i.e., the optimal current patterns, cannot be determined analytically for an arbitrary resistivity distribution. Isaacson [8] derived these patterns for some special resistivity distributions. Gisser $e t$ al. [2] proposed an iterative method to derive one optimal current pattern corresponding to the dominant eigensystem. However, their method requires lengthy acquisition time. In this paper, we developed both iterative and direct methods to determine a complete set of optimal current patterns for an arbitrary resistivity distribution.

\section{Methods to Determine Optimal Current PatTernS}

\section{A. Iterative Method}

For a $N$-electrode system, the matrix $\boldsymbol{D}$ has eigenvalues $\lambda_{0}, \lambda_{1}, \cdots, \lambda_{N-1}$ in a decreasing order, and corresponding eigenvectors $\boldsymbol{I}_{0}, \boldsymbol{I}_{1}, \cdots, \boldsymbol{I}_{N-1}$. Then the matrix can be decomposed into the following form:

$$
\boldsymbol{D}=\lambda_{0} \boldsymbol{I}_{0} \boldsymbol{I}_{0}^{T}+\lambda_{1} \boldsymbol{I}_{1} \boldsymbol{I}_{1}^{T}+\cdots+\lambda_{N-1} \boldsymbol{I}_{N-1} \boldsymbol{I}_{N-1}^{T} .
$$

Since $D$ depends on the real resistivity distribution which is an unknown, Gisser et al. [2] proposed an iterative method to

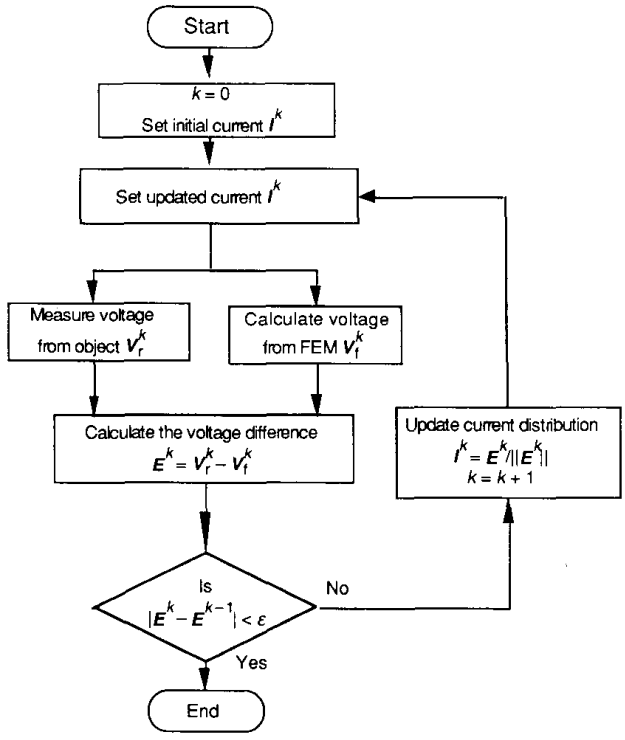

Fig. 2. The algorithm of Gisser et al. [2] performs $k$ iterations to yield a dominant optimal current pattern.

derive the dominant eigensystem, i.e., the largest eigenvalue with its eigenvector. Fig. 2 shows a flow chart of their algorithm. In our implementation of this algorithm, we set an initial current pattern, measure its voltage response from the real object, and calculate its voltage response for the estimated resistivity distribution. We then calculate and normalize the voltage difference, and use it as the updated current pattern. Eventually, the current pattern will converge to the eigenvector. This procedure requires no advance knowledge of the resistivity distribution and is numerically stable. However, it does require repetitive measurements from the real object.

After finding the dominant eigensystem $\lambda_{0}$ and $I_{0}$, we form another matrix $D^{\prime}$ as follows:

$$
\begin{gathered}
\boldsymbol{D}^{\prime}=\boldsymbol{D}-\lambda_{0} \boldsymbol{I}_{0} \boldsymbol{I}_{0}^{T}=\lambda_{1} \boldsymbol{I}_{1} \boldsymbol{I}_{1}^{T}+\lambda_{2} \boldsymbol{I}_{2} \boldsymbol{I}_{2}^{T} \\
+\cdots+\lambda_{N-1} \boldsymbol{I}_{N-1} \boldsymbol{I}_{N-1}^{T} .
\end{gathered}
$$

Then using the algorithm again with $\boldsymbol{D}^{\prime}$ substituted for $\boldsymbol{D}$, we obtain the dominant eigensystem of $\boldsymbol{D}^{\prime}$, i.e., $\lambda_{\mathbf{l}}$ and $\boldsymbol{I}_{1}$. We repeat the above procedure to obtain the rest of the desired current distributions.

In vivo applications of the algorithm have two major drawbacks.

1) The acquisition time is lengthy. For our 32 electrode hardware system, the acquisition time to obtain one set of voltage measurements is $24 \mathrm{~ms}$. We found that more than ten iterations are necessary to obtain a converged current projection with 12 bit resolution. Therefore, the acquisition time for one optimal current projection will be $240 \mathrm{~ms}$. For 31 patterns, the total acquisition time is $7.2 \mathrm{~s}$.

Many factors can cause resistivity change during this dataacquisition interval. If we image the human thorax, the heart rate is about one beat/s, and cardiovascular ejection time is about $250 \mathrm{~ms}$. The blood has a lower resistivity than 
other tissues in the chest, and thus ejection will change the resistivity distribution during the cycle of data collection. The pulmonary ventilation cycle lasts about $8 \mathrm{~s}$. The air exchange and morphological changes also result in resistivity change. Any of these changes during the data-collection period will result in distorted measurements and a blurred image.

2) It is difficult to update optimal current patterns. Hua [5] showed that updating optimal currents every few reconstruction iterations improves the image quality in an iterative reconstruction algorithm. The reconstruction time depends upon the reconstruction algorithm, computational method and the computer facility. By using the sparse matrix technique to compute the voltages for the FEM model with 224 elements and 201 nodes to minimize the computing time [11], we can reconstruct one image update on a Macintosh II with a math coprocessor in $4 \mathrm{~min}$. Goble et al. [3] use a Cray mainframe supercomputer to reduce the reconstruction time. However, the communication delay between the mainframe and the datacollection facility is lengthy. In order to update the optimal currents, we need to attach the instrument to a patient, and the resisitivity change during the reconstruction period also introduces measurement errors.

Fig. 3 shows a data-synthesis-based adaptive system which overcomes the above problems. We apply a complete set of independent current patterns to the object, measure their voltage responses, and store these data in the independent voltage bank. In order to derive optimal current patterns we can synthesize any current pattern's voltage response based on the principle of superposition instead of measuring voltages from the instrument, because the relationship between voltage and current is linear. That is, since

$$
\boldsymbol{I}=\sum_{i=0}^{N-1} a_{i} \boldsymbol{I}_{i}
$$

where $a_{i}$ is a scalar coefficient, and $\boldsymbol{I}_{0}$ to $\boldsymbol{I}_{N-1}$ are current bases defined as a complete set of independent current patterns with $\left\|\boldsymbol{I}_{i}\right\|=1$, the resulting voltage response can be synthesized as

$$
\boldsymbol{V}=\boldsymbol{R} \boldsymbol{I}=\sum_{i=0}^{N-1} a_{i} R \boldsymbol{I}_{i}=\sum_{i=0}^{N-1} a_{i} \boldsymbol{V}_{i}
$$

where $\boldsymbol{V}_{i}=R \boldsymbol{I}_{i}$, the voltages response of a current base $\boldsymbol{I}_{i}$.

In this way, we can remove the instrument from the object as soon as we finish the present independent measurements and use reconstruction algorithms to obtain the image later, making the system more practical and feasible. As we explain in the next section, there are $(N-1)$ independent projections for an $N$-electrode system. Thus the total acquisition time $=24 \mathrm{~ms}$ $\times 31=744 \mathrm{~ms}$ for our 32 electrode system, which is one tenth of the acquisition time for the original iterative methods.

\section{B. Direct Method}

The resistance matrix $\boldsymbol{R}(\rho)$ is a function of the real resistivity distribution $\rho$ and is difficult to obtain analytically without knowing the resistivity distribution $\rho$. Here we developed experimental methods to determine the resistance matrix $\boldsymbol{R}(\rho)$ for an unknown distribution $\rho$ through physical measurements.

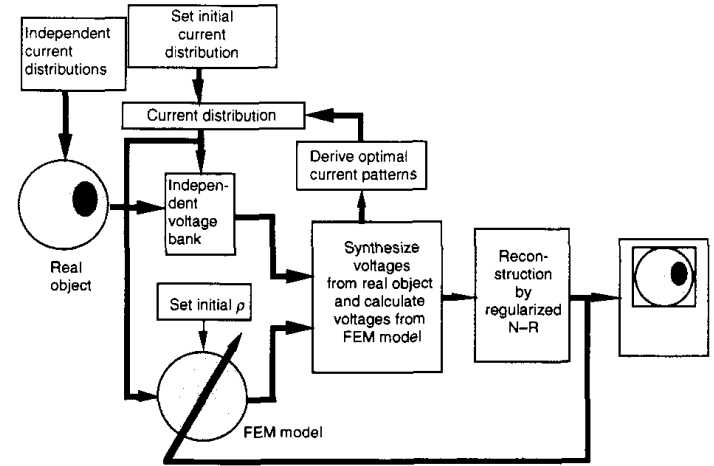

Fig. 3. We make a set of independent measurements and store them in a voltage bank. We can then obtain a voltage response for a given current pattern by synthesizing data from the voltage bank. This enables us to remove the test object from an EIT instrument once the independent measurements are made.

Then the $D$ matrix and its eigensystem can be determined explicitly.

Fig. 4 shows that we modeled the current and voltage relationship of an object in an $N$-electrode EIT system into an $N$-terminal network. The circuitry ground is used as a reference. Let vector $I=\{I(k)\}, k=0, \cdots, N-1$, denotes the currents we inject into the object through the $N$ electrodes, vector $\boldsymbol{V}=\{V(k)\}, k=0, \cdots, N-1$, denotes the voltages we measured from the $N$ electrodes, and matrix $\boldsymbol{R}(\rho)$ denotes the resistance between current and voltage. By Ohm's law, the relation among voltage $\boldsymbol{V}$, current $\boldsymbol{I}$, and resistance $\boldsymbol{R}(\rho)$ is

$$
\boldsymbol{V}=\boldsymbol{R}(\rho) \boldsymbol{I}
$$

i.e.,

$$
\begin{gathered}
{\left[\begin{array}{l}
V(0) \\
V(1) \\
V(2) \\
\cdots \\
V(N-1)
\end{array}\right]} \\
=\left[\begin{array}{llll}
R_{00} & R_{01} & R_{0 N-2} & R_{0 N-1} \\
R_{10} & R_{11} & R_{1 N-2} & R_{1 N-1} \\
R_{20} & R_{21} & R_{2 N-2} & R_{2 N-1} \\
\cdots & \cdots & \cdots & \cdots \\
R_{N-10} & R_{N-11} & R_{N-1 N-2} & R_{N-1 N-1}
\end{array}\right]\left[\begin{array}{l}
I(0) \\
I(1) \\
I(2) \\
\cdots \\
I(N-1)
\end{array}\right](7)
\end{gathered}
$$

where $R_{i j}$ denotes the transfer resistance between terminal $i$ and $j$. The resistance matrix $\boldsymbol{R}$ is a generalized inverse of the conductance matrix $Y$ satisfying equation $Y V=I$ from the node analysis of the network. Therefore, its element $R_{i j}$ has a sign (positive or negative) that is determined by the reference directions of current and voltage.

The human body is a passive continuous medium at $50 \mathrm{kHz}$. Since there are no controlled sources in the medium, the resistance network is reciprocal, i.e., the matrix $R$ is symmetric 


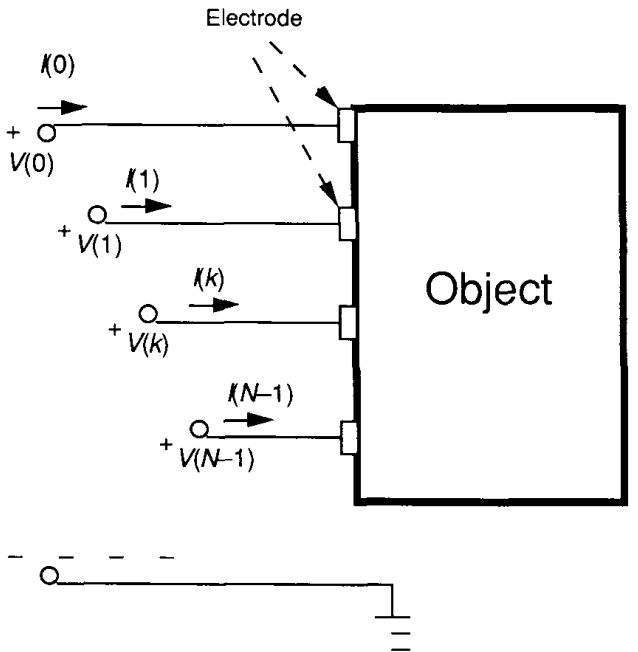

Fig. 4. We model the relations among currents and voltages in an $\mathrm{N}$-electrode system using an $\mathrm{N}$-terminal network.

as follows:

$$
\boldsymbol{R}_{i j}=\boldsymbol{R}_{j i}
$$

The continuous medium also ensures that none of the terminals is isolated from the rest of the network. Thus, the matrix is indefinite and satisfies the following:

$$
\sum_{i=0}^{N-1} \boldsymbol{R}_{i j}=c \text { for } j=0 \text { to } \mathrm{N}-1
$$

where $c$ is any constant.

Kirchhoff's current law states that

$$
\sum_{k=0}^{N-1} \boldsymbol{I}(k)=0
$$

Equations (7) to (10) result in

$$
\sum_{k=0}^{N-1} \boldsymbol{V}(k)=0
$$

In a physical measurement, we measure the differential voltages between electrodes. We can use (11) to adjust the measured differential voltages so that the resulting voltages have the same reference as the estimated voltages by FEM.

Note that due to the relationship shown in (9), there are only $(N-1)$ eigenvalues and eigenvectors for the resistance matrix $R$, and thus also the matrix $D$. By injecting a set of independent current bases $\boldsymbol{I}_{i}, i=0, \cdots, N-2$, we can measure the resulting voltages $\boldsymbol{I}_{i}, i=0, \cdots, N-2$. We use (7)-(10) to uniquely determine all the elements of the resistance matrix $\boldsymbol{R}$.

Similarly, we can inject the same set of current bases into our FEM, in which we model the electric field distribution using a finite element mesh with a total of $M$ nodes [5]. By enforcing the governing equation of the electric field, i.e., the Poisson equation, we generate a set of linear equations $\boldsymbol{Y}_{F}(\hat{\rho}) \boldsymbol{V}_{F}=\boldsymbol{I}_{F}$ where $\boldsymbol{Y}_{F}$ is a $M$ by $M$ matrix whose values depend on the resistivity distribution $\hat{\rho}$ and the geometry of the elements, $V_{F}$ is a $M$ by 1 vector that denotes node voltages, and $I_{F}$ is a $M$ by 1 vector that denotes external current loads for each node. For each injected current base $I_{i}$ through the electrodes, we can map its values into the corresponding node current vector $I_{F}$, and solve the linear equations to obtain the node voltage vector $V_{F}$. We then extract the node voltages corresponding to the electrode location to yield the electrode voltages $\boldsymbol{V}_{i}$. As a result, we can also solve for $\boldsymbol{R}(\hat{\rho})$ using (7)-(10), thus explicitly determine the $D$ matrix.

We can use many methods to obtain the eigensystem of this symmetric $D$ matrix: the QR algorithm, singular value decomposition (SVD), the Jacobi method, etc. [4]. We chose the SVD method because it is stable and straightforward to obtain. SVD permits decomposition of a matrix $D$ into orthogonal matrices $\boldsymbol{U}, \boldsymbol{V}$ and a diagonal matrix $\boldsymbol{L}$ so that

$$
U^{T} \boldsymbol{D} \boldsymbol{V}=\boldsymbol{L}
$$

$\boldsymbol{L}=\operatorname{diag}\left\{\lambda_{0} \lambda_{2}, \cdots, \lambda_{N-1}\right\}$ are the eigenvalues of $\boldsymbol{D}$, and $U$ and $V$ are the eigenvectors matrices. Once we obtain the optimal current patterns and the resistance matrix $\boldsymbol{R}$, the corresponding voltages can be solved through a matrix multiplication.

\section{DIFFERENT CURRENT BASIS}

To determine the optimal current patterns, both the synthesisbased iterative method and the direct method require the injection of a set of independent current bases into the object of interest: the former for synthesizing the voltage response of a given current distribution, the latter for determining the resistance matrix. Many patterns can be used as independent current bases. In this section, we examine three types of bases in detail: Fourier, diagonal, and neighboring.

\section{A. Fourier-Based Method}

Let $I(k)$ be the current flowing through the $k$ th electrode in a $N$-electrode system, and let $W_{N}$ be the principal $N$ th root of unity as follows:

$$
W_{N}=e^{j 2 \pi / N} .
$$

The current pattern can be represented as a function of higher harmonics:

$$
I(k)=\frac{1}{N} \sum_{n=0}^{N-1} C(n) W_{N}^{k n} \quad k=0,1, \cdots, N-1
$$

where $C(n)$ is the discrete Fourier transform (DFT) defined as

$$
C(n)=\sum_{k=0}^{N-1} I(k) W_{N}^{-k n} .
$$


If $C=[C(0), \cdots, C(N-1)]^{T},(15)$ can be expressed in a matrix form as

$$
C=F I
$$

where $\boldsymbol{F}$ is the $N$ by $N$ matrix with elements

$$
F_{n k}=W_{N}^{-k n}, \quad 0 \leq k, n \leq N-1
$$

1) Voltage Synthesis Method: A current pattern can thus be expressed as

$$
\boldsymbol{I}=\frac{1}{N} \boldsymbol{F}^{*} \boldsymbol{C}
$$

where $\boldsymbol{F}^{*}$ denotes complex conjugate of $\boldsymbol{F}$. Each column of the $F^{*} / \sqrt{N}$ matrix forms a current base $I_{i}$ and $C(i) / \sqrt{N}$ forms the coefficient $a_{i}$ in (4). Therefore, the resulting voltage is

$$
\boldsymbol{V}=\boldsymbol{R I}=\frac{1}{N} \boldsymbol{R} \boldsymbol{F}^{*} \boldsymbol{C}=\frac{1}{N} \boldsymbol{V}_{f} \boldsymbol{C}
$$

where

$$
\boldsymbol{V}_{f}=\boldsymbol{R} \boldsymbol{F}^{*}
$$

The $V_{f}$ matrix represents the voltage responses of current $\boldsymbol{F}^{*}$. In order to synthesize a voltage response, we need to calculate the Fourier coefficients according to (15), and calculate the voltage response according to (16).

2) Direct Method to Determine $\boldsymbol{R}: \boldsymbol{F}$, the matrix representation of the DFT, is an orthogonal matrix since

$$
\left[\boldsymbol{F}^{*} \boldsymbol{F}\right]_{m n}=N \delta(m-n)
$$

or

$$
\boldsymbol{F}^{*} \boldsymbol{F}=N \boldsymbol{I}
$$

We multiply $\boldsymbol{F}$ by both sides of (17) to obtain

$$
\boldsymbol{V}_{f} \boldsymbol{F}=\boldsymbol{R} \boldsymbol{F}^{*} \boldsymbol{F}=N \boldsymbol{R},
$$

i.e.,

$$
\boldsymbol{R}=\frac{1}{N} \boldsymbol{V}_{f} \boldsymbol{F}
$$

The resistance matrix $R$ can be obtained by a matrix multiplication of the voltage response $V_{f}$ with the currents $\boldsymbol{F}$.

The current distribution when $k=0$ is all 1 , which cannot be physically applied to an object since its sum is not zero. However, its voltage response is zero according to (7), (8), (9), and (11). The derivation of a resistance matrix in (20) requires the injection and measurement of 62 current patterns and a matrix multiplication.

\section{B. Diagonal-Based Method}

In this method, we select one electrode as a reference and choose another electrode as an active electrode to inject current. We then alter the active electrode until the rest of the electrodes have been used as active electrodes. The current bases are formed as $\boldsymbol{I}_{0}=\left[1 / \sqrt{2}, 0,0, \cdots,-1 / \sqrt{2} T, \boldsymbol{I}_{1}=\right.$ $\left[0.1 / \sqrt{2}, 0, \cdots,-1 / \sqrt{2} T, \boldsymbol{I}_{N-2}=[0, \cdots, 0,1 / \sqrt{2},-1\right.$ $/ \sqrt{2}]^{T}$.

1) Voltage Synthesis Method: An arbitrary current pattern can be represented as

$$
\begin{aligned}
\boldsymbol{I} & =[I(0), \cdots, I(N-1)]^{T} \\
& =\sqrt{2}\left\{I(0) \boldsymbol{I}_{0}+\cdots+I(N-2) \boldsymbol{I}_{N-2}\right\}=\sqrt{2} \sum_{k=0}^{N-2} I(k) \boldsymbol{I}_{i} .
\end{aligned}
$$

Therefore, its voltage response can be calculated as

$$
\boldsymbol{V}=\boldsymbol{R}(\rho) \boldsymbol{I}=\sqrt{2} \sum_{k=0}^{N-2} I(k) \boldsymbol{V}_{i}
$$

where $\boldsymbol{V}_{i}=\boldsymbol{R}(\rho) \boldsymbol{I}_{i}$, the measured voltages of the diagonal currents $\boldsymbol{I}_{i}$. Thus the estimated voltage for a given current is a weighted function of the premeasured voltages from independent currents.

2) Direct Method to Determine R: We assemble the measured voltages into a new matrix as shown in (23) at bottom of the page. Adding all the components in the $i$ th row and using (9), we obtain

$$
\begin{aligned}
& \sum_{j=0}^{N-1} V^{\prime}(i, j)=\frac{1}{\sqrt{2}}\left\{\left(R_{i 0}+\cdots+R_{i N-2}\right)-(N-1) R_{i N-1}\right\} \\
& =\frac{1}{\sqrt{2}}\left\{\left(R_{i 0}+\cdots+R_{i N-1}\right)-N R_{i N-1}\right\}=\frac{c-N R_{i N-1}}{\sqrt{2}}
\end{aligned}
$$

$$
\begin{aligned}
\boldsymbol{V}^{\prime} & =\left[\boldsymbol{V}_{0}, \cdots, \boldsymbol{V}_{i}, \cdots, \boldsymbol{V}_{N-2}\right] \\
& =\frac{1}{\sqrt{2}}\left[\begin{array}{lll}
R_{00}-R_{0 N-1} & R_{01}-R_{0 N-1} & R_{0 N-2}-R_{0 N-1} \\
R_{10}-R_{1 N-1} & R_{10}-R_{1 N-1} & R_{1 N-2}-R_{1 N-1} \\
\cdots & \cdots & \cdots \\
R_{N-20}-R_{N-2 N-1} & R_{N-21}-R_{N-2 N-1} & R_{N-2 N-2}-R_{N-2 N-1} \\
R_{N-10}-R_{N-1 N-1} & R_{N-11}-R_{N-1 N-1} & R_{N-1 N-2}-R_{N-1 N-1}
\end{array}\right] .
\end{aligned}
$$


Thus, we may decompose the $n$th column in the $R$ matrix by

$$
R_{i N-1}=-\frac{\sqrt{2}}{N} \sum_{j=0}^{N-1} V^{\prime}(i, j)+\frac{c}{N} .
$$

We obtain the rest of the elements of $\boldsymbol{R}$ by adding $R_{i N-1}$ to all the columns for all rows in matrix $\boldsymbol{V}^{\prime}$. It is easy to prove that the resistance matrix $R$ determined by any selection of $c$ produces that same voltages for a given current pattern due to its indefiniteness. Therefore, we select $c=0$ to simplify computation.

\section{Neighboring-Based Method}

Barber and Brown [1] sequentially used a single current generator to inject current through a pair of adjacent electrodes and measure the voltages from all the other electrodes. The corresponding current bases are $\boldsymbol{I}_{0}=[1 / \sqrt{2},-1 / \sqrt{2}, 0, \cdots, 0]^{T}, \boldsymbol{I}_{2}=[0,1 / \sqrt{2},-1 / \sqrt{2}$, $0, \cdots, 0]^{T}, \boldsymbol{I}_{N-2}=[0, \cdots, 0,1 / \sqrt{2},-1 / \sqrt{2}]^{T}$.

1) Voltage Synthesis Method: An arbitrary pattern can be represented as

$$
\begin{aligned}
\boldsymbol{I}= & \sqrt{2}\left\{I(0) \boldsymbol{I}_{0}+(I(0)+I(1)) \boldsymbol{I}_{1}+\cdots\right. \\
& \left.+(I(0)+\cdots+I(N-2)) \boldsymbol{I}_{N-2}\right\} \\
= & \sqrt{2} \sum_{i=0}^{N-2}\left\{\sum_{k=0}^{i} I(k)\right\} \boldsymbol{I}_{i} .
\end{aligned}
$$

Therefore, its voltage response can be calculated as

$$
\boldsymbol{V}=\sqrt{2} \sum_{i=0}^{N-2}\left\{\sum_{k=0}^{i} I(k)\right\} \boldsymbol{V}_{i}
$$

where $\boldsymbol{V}_{i}=\boldsymbol{R}(\rho) \boldsymbol{I}_{i}$, the measured voltages of the neighboring currents $\boldsymbol{I}_{i}$.

2) Direct Method to Determine R: The direct method to determine $\boldsymbol{R}$ is straightforward. However we found that the synthesized voltages by this method were very noisy. Thus the direct method to determine $\boldsymbol{R}$ is not significant and is omitted in this discussion.

\section{RESULTS AND DISCUSSIONS}

\section{A. Comparison of Voltage-Synthesis Methods}

Equation (4) shows that $\boldsymbol{V}=\sum_{i=0}^{N-1} a_{i} \boldsymbol{V}_{i}$. Since the measured voltage $\boldsymbol{V}_{i}$ contains both the ideal signal $\boldsymbol{S}_{i}$ and noise $\boldsymbol{N}_{i}$, we obtain

$$
\boldsymbol{V}_{i}=\boldsymbol{S}_{i}+\boldsymbol{N}_{i}
$$

Thus,

$$
\boldsymbol{V}=\sum_{i=0}^{N-1} a_{i}\left(\boldsymbol{S}_{i}+\boldsymbol{N}_{i}\right)=\sum_{i=0}^{N-1} a_{i} \boldsymbol{S}_{i}+\sum_{i=0}^{N-1} a_{i} \boldsymbol{N}_{i}
$$

where the first term is the measured signal and the second is the error in the synthesized voltage.
If we assume the error is a zero-mean Gaussian noise, and the variance for each $\boldsymbol{N}_{i}$ is equal to $\sigma^{2}$ and uncorrelated, the variance $\sigma_{v}^{2}$ for the error in $\boldsymbol{V}$ is given by

$$
\frac{\sigma_{v}^{2}}{\sigma^{2}}=\sum_{i=0}^{N-1} a_{i}^{2}
$$

Thus the noise level is proportional to the norm of the coefficient vector used in different current bases. We can prove that the variances in the Fourier, diagonal-, and neighboringbased methods are

$$
\begin{gathered}
\frac{\sigma_{v}^{2}}{\sigma^{2}}=\sum_{k=0}^{N-2} I(k)^{2}, \quad 2 \sum_{k=0}^{N-2} I(k)^{2}, \\
\text { and } 2 \sum_{j=0}^{N-2}\left(\sum_{k=0}^{j} I(k)\right)^{2}
\end{gathered}
$$

respectively. Thus, the error level is a function of current distribution. The Fourier-based method has less error than the diagonal-based method.

The actual noise source, however, may not follow an ideal additive Gaussian distribution. A noise in the current source may result in multiplicative noise. Thus the above derivation can only provide some guidelines for a statistical measure.

We studied the accuracy of different voltage-synthesis methods through experiments using our 32-electrode dataacquisition system with 12 bit resolution. For a given current pattern, we measured the voltage responses from a physical phantom 16 times and averaged them to generate a measured voltage $V_{m}$. We then measured voltages for different independent current patterns and used the above methods to synthesize the voltages $\boldsymbol{V}_{s}$. We defined the error-to-signal ratio as $e=\left\|\boldsymbol{V}_{m}-\boldsymbol{V}_{s}\right\| /\left\|\boldsymbol{V}_{m}\right\|$ where $\|\boldsymbol{x}\|$ denotes the norm of a vector $\boldsymbol{x}$. In order to estimate the system noise level $N_{e}$, we calibrated the data-acquisition hardware and set all current generators. The resulting voltages were mainly the noise due to the high-frequency leakage by stray capacitance and quantization error by the analog-to-digital converter in a voltmeter and the digital-to-analog converter in a current source. We defined the noise-to-signal ratio as $\left\|N_{e}\right\| /\left\|\boldsymbol{V}_{m}\right\|$ so that we can compare the synthesized voltage error with the system noise.

We injected the following current patterns.

1) Symmetric pulse (SP)

$$
\begin{aligned}
I(n) & =1 / \sqrt{2} & & \text { when } n=7 \\
& =-1 / \sqrt{2} & & \text { when } n=23 \\
& =0 & & \text { otherwise. }
\end{aligned}
$$

2) Fundamental harmonics (FH)

$$
I(n)=\sin (2 \pi n / N) / \sqrt{N / 2} \quad n=0 \text { to } N-1 .
$$

3) Higher harmonics (HH)

$$
I(n)=\sin (2 \pi 4 n / N) / \sqrt{N / 2} \quad n=0 \text { to } N-1 .
$$


TABLE I

THE ERRor of DifFerent Voltage-Synthesis Methods for Different Current Patterns in Percent

\begin{tabular}{ccccc}
\hline \multirow{2}{*}{$\begin{array}{l}\text { Injected } \\
\text { current } \\
\text { pattern }\end{array}$} & \multicolumn{2}{c}{ Error-to-signal ratio (\%) } & $\begin{array}{l}\text { Noise-to- } \\
\text { signal } \\
\text { ratio }(\%)\end{array}$ \\
\cline { 2 - 5 } & Neighboring & Diagonal & Fourier & 0.0305 \\
SP & 0.3813 & 0.0380 & 0.0344 & 0.0073 \\
FH & 0.5053 & 0.0087 & 0.0070 & 0.0043 \\
HH & 1.0278 & 0.0397 & 0.0491 & 0.0308 \\
UDP & 0.8517 & 0.0323 & 0.0336 & 0.0303 \\
\hline
\end{tabular}

4) Unsymmetrical dual-pulses (UDP)

$$
\begin{aligned}
I(n) & =1 / \sqrt{4} & & \text { when } n=7 \text { and } 8 \\
& =-1 / \sqrt{4} & & \text { when } n=16 \text { and } 23 \\
& =0 & & \text { Otherwise. }
\end{aligned}
$$

Table I summarizes the experimental results. It shows that the noise is about $0.03 \%$ of the signal level, which is about 1 bit of resolution in the 12 bit acquisition system. The ratios of the error-to-noise (error-to-signal ratio/noise-to-signal ratio) range from $12-28$ for neighboring, 1.1-2.0 for diagonal, and 1.1-1.6 for Fourier methods. The Fourier method introduces an error on the order of the noise level, and is the most accurate method.

What affects the accuracy of different synthesis methods is the measurement error in the hardware system. For the neighboring-based method, the current density of a current base is high in the vicinity of one pair of adjacent current electrodes. The voltage at other electrodes is small, thus the signal-to-noise ratio (SNR) is small and this method has a large error level. The current density in the diagonal method is more uniform than that in the neighboring method. Thus the resulting voltages have a much larger SNR than those from the neighboring method. The synthesized voltages are more accurate than those from the neighboring method. The current densities in the Fourier method are more uniform than those in the neighboring and diagonal methods. Thus resulting voltages from the Fourier method have a much larger SNR than those from other methods and the Fourier method has the most accurate results.

\section{B. Comparison of Optimal Currents}

We put a $5 \mathrm{~cm}$ glass beaker into a $30 \mathrm{~cm}$ diameter tank filled with $100 \Omega$-cm saline solution. Fig. 5 shows the reconstructed image using optimal current patterns derived from both direct and iterative methods for different types of current bases. The images from optimal currents by direct methods are better than those by the iterative methods. The neighboring-based method does not produce a quality image due to the poor SNR shown in Table I. Both the Fourier-based and the diagonal-based methods produce satisfactory results.

We further compare the performance of Fourier current bases and diagonal current bases and diagonal current bases through forward evaluation. We injected into the phantom a set of current patterns $\boldsymbol{I}_{k}(i)=\cos (2 \pi k i / 32) ; i=1, \cdots, 32, k=$ $1, \cdots, 16$, and measured their voltage responses $V_{m}$. We then synthesized the voltages $V_{s}$ using the Fourier-based and the

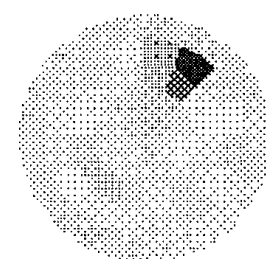

(a)

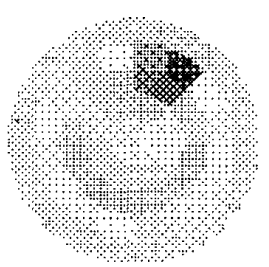

(c)

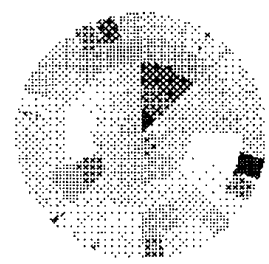

(e)

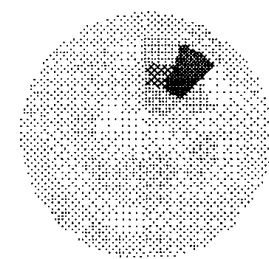

(b)

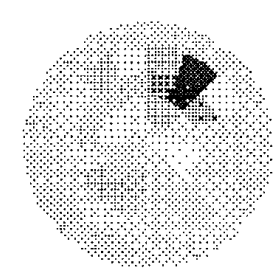

(d)

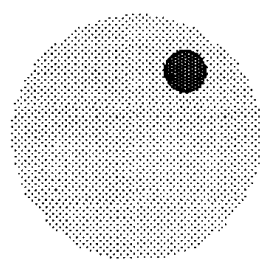

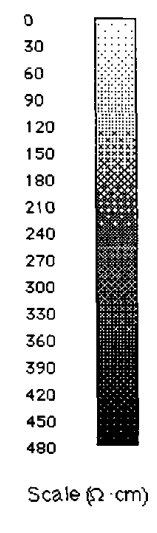

Fig. 5 . A $5 \mathrm{~cm}$ glass beaker was placed in a $30 \mathrm{~cm}$ diameter tank filled with $100 \Omega \cdot \mathrm{cm}$ saline. The reconstructed image using (a) the Fourier-based direct method, (b) the diagonal-based direct method, (c) the Fourier-based iterative method, (d) the diagonal-based iterative method, and (e) the neighboring-based iterative method. (f) The original image.

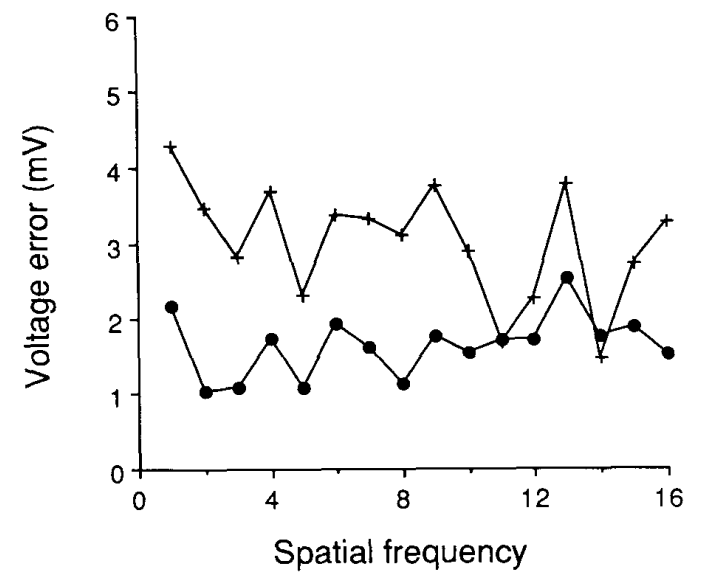

Fig. 6. The synthesized voltage error for the Fourier-based direct method $(\bullet)$ is smaller than that of the diagonal-based direct method $(+)$.

diagonal-based direct methods respectively. We calculated the absolute error between the measured and synthesized voltages, i.e., $\left\|\boldsymbol{V}_{m}-\boldsymbol{V}_{s}\right\|$. Fig. 6 shows that the Fourier-based direct method has a smaller error than the diagonal-based method, especially for low spatial frequencies, which concludes that the Fourier-based direct method is the most accurate method among the investigated methods.

Using the Fourier-based direct method, we can determine 
the resistance matrix $\boldsymbol{R}_{f}$ and the corresponding optimal currents and voltages. The matrix $\boldsymbol{R}_{f}$, however, is not the most accurate one, since the voltage responses of a set of Fourier current patterns do not always have the best SNR's for an arbitrary resistivity distribution. Thus, in an application where resistivity distribution change is slow with respect to the data-acquisition speed, we can inject these current patterns into the subject again to measure their voltage responses. Since the injected currents are a set of eigenvectors which are orthogonal, we can use a simple matrix multiplication to determine a new resistance matrix $\boldsymbol{R}_{n}$ based on these measurements. The new resistance matrix should be more accurate since the injected current patterns are closer to the ideal optimal currents, and the resulting voltages have better SNR's. However, for in vivo applications the physiological changes may prevent us from using this approach.

The numerical round-off error and the noise in the dataacquisition system introduce errors in the optimal current pattern generation for the iterative methods. These errors are accumulated in the generation of the next optimal current pattern, and a small stopping error and accurate representation of the variables are necessary. The direct methods do not have this error accumulation and thus perform better than the iterative methods.

\section{Single Versus Multiple Current Generator System}

There are generally two types of EIT: single and multiple (mostly 32) current generators. The multiple generator system can inject optimal current patterns while the single generator can only provide a single current. Use of optimal current patterns can achieve better distinguishability and produce better images.

The hardware design of the multiple-generator system, however, is much more complicated than that of the singlegenerator system. In addition to more components, the multiple generator system requires good balance among all generators of output impedance, amplitude, and phase. For a carrier frequency of $50 \mathrm{kHz}$, stray capacitance causes imbalance problems. The balancing would be even more difficult for three-dimensional imaging where 64 or more generators might be required to achieve reasonable spatial resolution. The diagonal-based method described above requires one current generator to inject current for each projection. Table I shows that the voltages thus synthesized have the same order of accuracy as the Fourier method which requires multiple generators to provide the desired current patterns. The reconstructed image in Fig. 6 has an image comparable to the one from the Fourier-based method. Thus we can generate optimal current patterns using a single generator system such as the Sheffield data-acquisition system [1] an achieve comparable resolution to a multiple generator system.

This paper described methods to generate optimal current patterns for a 2-D study. These methods are also applicable to 3-D studies since the linear relationship between current and voltage holds true for both cases.

\section{CONCLUSION}

We have shown that the voltage response for a given current pattern can be synthesized from preset independent measurements with the introduced error being of the same order as the system noise. The optimal current patterns derived by direct methods are better than those by iterative methods. The Fourier-based method produces the most accurate synthesized voltages and optimal current patterns. We have shown that we can use the single-channel current generator and the diagonal method to generate an optimal current pattern and its voltage response, and achieve slightly less accurate but comparable results with that of a multiple-generator system.

\section{REFERENCES}

11] D. C. Barber and B.H. Brown, "Recent developments in applied potential tomography," in Information Processing in Medical Imaging, S. L. Bacharach, Ed. Dordrecht: Matrinus Nijhoff, 1986.

[2] D. G. Gisser, D. Isaacson, and J. C. Newell, "Current topics in impedance imaging," Clin. Phys. Physiol. Meas., vol. 8, suppl. A, pp. 39-46, 1987.

[3] J.C. Goble and T.D. Gallagher, "A distributed architecture for medical instrumentation: An electrical current computed tomograph," in Proc. Annu. Int. Conf. IEEE Eng. Med. Biol. Soc., vol. 10, pp. 285-286, 1988.

[4] G. H. Golub and C. F. Van Loan, Matrix Computation. Baltimore, MD: The Johns Hopkins Univ. Press, 1983.

[5] P. Hua, "Modeling and reconstruction methods for electrical impedance tomography," Ph. D. dissertation, Dep. Elec. Comp. Eng., Univ. of Wisconsin, Madison, WI, 1990.

[6] P. Hua, J.G. Webster, and W.J. Tompkins, "A regularised electrical impedance tomography reconstruction algorithm," Clin. Phys. Physiol. Meas., vol. 8, suppl. A, pp. 137-141, 1988.

[7] P. Hua and E.J. Woo, "Reconstruction algorithms," in Electrical Impedance Tomography, J.G. Webster, Ed. Bristol, England: Adam Hilger, 1990, pp. 97-137.

[8] D. Isaacson, "Distinguishability of conductivities by electric current computed tomography," IEEE Trans. Med. Imaging, vol. MI-5, pp. $91-95,1986$.

[9] R. J. Lytle and K.A. Dines, "An impedance camera: A system for determining the spatial variation of electrical conductivity," Lawrence Livermore Lab., Livermore, CA, 1978.

[10] S. Bhat, "Clinical applications," in Electrical Impedance Tomography, J. G. Webster, Ed. Bristol, England: Adam Hilger, 1990, pp. 175-200

[11] E. J. Woo, P. Hua, W. J. Tompkins, and J. G. Webster, "A finite element model with node renumbering for adaptive impedance imaging," in Proc. Annu. Int. Conf. IEEE Eng. Med. Biol. Soc., vol. 10, pp. 277-278, 1988.

[12] T.J. Yorkey, J. G. Webster, and W. J. Tompkins, "Comparing reconstruction algorithms for electrical impedance tomography," IEEE Trans. Biomed. Eng., vol. BME-34, pp. 843-852, 1987. 